\title{
Non-ribosomal insights into ribosomal P2 protein in Plasmodium falciparum-infected erythrocytes
}

\author{
Sudipta Das ${ }^{1}$, Bhaskar Roy ${ }^{1}$, and Saswata Chakrabarty ${ }^{1}$ \\ ${ }^{1}$ CSIR-Indian Institute of Chemical Biology
}

April 9, 2021

\begin{abstract}
The enormous complexity of the eukaryotic ribosome has been a real challenge in unlocking the mechanistic aspects of its amazing molecular function during mRNA translation and many non-canonical activities of ribosomal proteins in eukaryotic cells. While exploring the uncanny nature of ribosomal P proteins in malaria parasites Plasmodium falciparum, the 60S stalk ribosomal P2 protein has been shown to get exported to the infected erythrocyte (IE) surface as an SDS resistant oligomer during the early to mid trophozoite stage. Inhibiting IE surface P2 either by monoclonal antibody or through genetic knockdown resulted in nuclear division arrest of the parasite. This very strange and serendipitous finding has led us to explore more about un-canonical cell biology and structural involvement of P2 protein in Plasmodium in the search for a novel biochemical role during parasite propagation in the human host.
\end{abstract}

Non-ribosomal insights into ribosomal P2 protein inPlasmodium falciparum-infected erythrocytes

Sudipta Das ${ }^{1 \#,}$ Bhaskar Roy ${ }^{1}$, Saswata Chakrabarty ${ }^{1}$

Asymmetric Cell Division Laboratory, Room No. 219, Division of Infectious Disease and Immunology, CSIR-Indian Institute of Chemical Biology, 4, Raja S.C Mullick Road, Kolkata: 700032, India.

\# Correspondence: sudipta.das@iicb.res.in

\begin{abstract}
The enormous complexity of the eukaryotic ribosome has been a real challenge in unlocking the mechanistic aspects of its amazing molecular function during mRNA translation and many non-canonical activities of ribosomal proteins in eukaryotic cells. While exploring the uncanny nature of ribosomal $P$ proteins in malaria parasites Plasmodium falciparum, the 60S stalk ribosomal P2 protein has been shown to get exported to the infected erythrocyte (IE) surface as an SDS resistant oligomer during the early to mid-trophozoite stage. Inhibiting IE surface P2 either by monoclonal antibody or through genetic knockdown resulted in nuclear division arrest of the parasite. This very strange and serendipitous finding has led us to explore more about un-canonical cell biology and structural involvement of P2 protein in Plasmodium in the search for a novel biochemical role during parasite propagation in the human host.
\end{abstract}

Keywords

Malaria; Plasmodium falciparum; ribosomal P-protein; protein trafficking; protein oligomerization; Channel protein complex; Nuclear division; Cell division.

\section{Introduction}


Plasmodium falciparum is a unicellular eukaryotic protozoan parasite that causes malaria, a catastrophic disease in many developing countries. Malaria alone is responsible for millions of deaths annually across the globe. Drug resistance in malaria parasites is a serious issue hence emphasizing discovering new drug targets and inventing novel small molecules which can target multiple pathways together ensuring reduced chances of resistance is one of the main challenges in the current malaria research effort. To explore new anti-malaria drug targets, parasite ribosome can be a new focus of research interest.

The Ribosome is a protein-synthesizing nanomachine present in all living cells. In prokaryotes, ribosome comprises of 30S small subunit and 50S larger subunit whereas in eukaryotic cells 40S and 60S are the smaller and larger subunit respectively. The building blocks of a ribosome particle are mainly rRNA and proteins. These protein molecules are exclusively associated with the ribosome and primarily engaged in their ribosomal activities but many ribosomal proteins have been discovered to perform non-ribosomal functions at a distance in a different compartment of a cell (Volarevic et al., 2000; Wan et al., 2007; Wool IG., 1996; Jiménez-Díaz et al., 2013; Tchórzewski et al., 2003). In the 60S ribosomal subunit, a structural protuberance known as stalk, which is directly involved in the interaction of the elongation factors with the ribosome during mRNA translation. The stalk is a complex of five phosphorylated proteins (P-proteins), four small acidic proteins, and a larger protein that directly interacts with the rRNA at the GTPase center during protein synthesis (Remacha et al., 1995a). In eukaryotes, there are three types of P proteins, P0, P1, and $\mathrm{P} 2$, wherein Saccharomyces cerevisiae acidic $\mathrm{P} 1[?]$ and $\mathrm{P} 1 \beta$ interact with $\mathrm{P} 2 \beta$ and $\mathrm{P} 2[?]$ respectively to form [(P1[?]-P2 $\beta)-\mathrm{P} 0-(\mathrm{P} 1 \beta-\mathrm{P} 2[?])]$ pentameric stalk (Remote et al., 1995b). In-plant, an additional P protein, P3, has been discovered to be a part of plant ribosomal stalk (Kang et al., 2016). The stoichiometry of $\mathrm{P} 1$ and $\mathrm{P} 2$ differ in different organisms and found to be in a constant exchange between ribosome and cell cytoplasm (Zinker et al., 1976). In yeast and also in human cell lines, depletion of P2 leads to an instantaneous degradation of P1(Nusspaumer et al., 2000; Martinez-Azorin et al., 2008). In Saccharomyces cerevisiae, $\mathrm{P} 0$ null strain is lethal whereas $\mathrm{P} 1 / \mathrm{P} 2$ or $\mathrm{P} 1-\mathrm{P} 2$ null strains do survive but growth rate diminishes significantly indicating their essential nature in cell survival (Remacha et al., 1992; Santos et al., 1995; Rodríguez-Mateos et al., 2009). These uncanny properties of ribosomal P-proteins have sparked the enthusiasm to investigate whether P-proteins in eukaryotic apicomplexan human parasites (e.g; malaria) have any extra-ribosomal indispensable functions which can be targeted for better therapeutic interventions. In the malaria-endemic area, serum of malaria immune person has been detected with antibodies against anti-P. falciparum P0 protein quite extensively and exclusively (Lobo et al., 1994; Chatterjee et al., 2000; Singh et al., 2002). To explore the possibility of a novel anti-malaria intervention using P-proteins, in a differential immunoscreen, Plasmodium falciparum 60S stalk ribosomal protein P0 (PfP0) was identified as a protective protein and subsequently localized on merozoite surface possibly involved during red blood cell invasion as understood using growth inhibition assay (GIA) (Lobo et al., 1994). In P. falciparum ribosome, P0 interact with P1 and P2 to form the pentameric stalk [(P1-P2)-P0-(P1-P2)] (Hanson et al., 2004; Gonzalo et al., 2003; Santos et al., 1994; Francisco-Velilla et al., 2010) required in the GTPase elongation center (Uchiumi et al., 1992; Diaconu et al., 2005). While exploring eccentric properties of P0 protein in Plasmodium, a very puzzling phenotype of acidic ribosomal protein P2 was discovered (Das et al., 2012a). Ribosomal P2 protein of Plasmodium falciparum translocates to the infected RBC (iRBC) surface as an SDS resistant oligomer and appears to play a pleiotropic role at the late trophozoite/early schizogonic stage in iRBCs (Das et al., 2012a; Das et al., 2012b). In this mini-review, we summarize recent discoveries of Plasmodium P2 protein and highlight key insights and questions which need to be pondered to understand eccentric P2 biology in malaria parasites.

\section{Plasmodium parasites appear to require P2 on the infected red cell surface at the tropho- zoite/early schizogonic stage}

During the late trophozoite/early schizogonic stage, SDS resistant P2 oligomers have been localized on the surface of iRBCs (Das et al., 2012a). Blocking the accessibility of oligomeric P2 on the iRBCs surface using highly specific monoclonal antibodies resulted in nuclear division arrest of the parasite (Das et al., 2012a). In the arrested parasites, the blockage of import of fluorescently labeled lipid molecule, FM4-64 has been observed may be as a consequence of the degradation of lipid importing structures called tubovesicular network 
(TVN) (Das et al., 2012a; Lauer et al., 1997; Haldar et al., 2001; Tames et al., 2008). Washing off antibodies from the $P$. falciparum culture medium resulted in the reformation of TVN structures, the continuation of parasite nuclear division, and the completion of schizogonic processes of arrested parasites (Das et al., 2012a), indicating a potential non-ribosomal role (s) of oligomeric P2 protein on the iRBCs surface. The P2 oligomers on iRBCs surface appear to be SDS resistant homo/hetero tetrameric in nature (Das et al., 2012a; Das et al., 2012b) which did not resolve to a monomer in SDS containing reducing polyacrylamide gels, however, contrary to the iRBC surface, P2 protein in the parasite cytoplasm is predominantly present as a monomer indicating a possible role of SDS resistant oligomerization and oligomeric form of P2 on iRBC surface at trophozoite stage of parasite growth. Hence oligomeric P2 on the iRBCs surface appears to be required for the progression of the parasite growth and development. The indispensability was further confirmed as the P2 gene was found to be refractory to deletion in Plasmodium berghei (Das et al., 2012a; Bushell et al., 2017) and recently shown in Plasmodium falciparum (Zhang et al., 2018). Apart from the iRBC surface, P2 protein has also been localized on the surface of Plasmodium falciparum $(\mathrm{Pf})$ and tachyzoite surface of Toxoplasma gondii (Tg) as either monomer or as an oligomer and have been implicated for host cell invasion using growth inhibition assay (GIA) (Sudarsan et al., 2015).

3. P2 oligomerization in the parasite cytoplasm appears to be one of the prerequisite step for translocation to the host cells

Effector proteins in P. falciparum -infected iRBCs translocate to the host cells and render the host cell membrane rigid and promiscuous for solutes and other macromolecules (Goldberg et al., 2010; Hiller et al., 2004; Russo et al., 2010). A well-defined class of export proteins possesses a Plasmodium export element (PEXEL) or vacuolar targeting signal, a pentapeptide consensus sequence (RxLxE/Q/D) located 25-30 amino acids downstream of the host-targeting signal sequence (Marti et al., 2004; Spillman et al., 2015; Desai et al., 2012; Desai et al., 2014a). The cleavage of the PEXEL motif by the ER-resident aspartic proteases, plasmepsin V is required to destine the export proteins to the host cells (Russo et al., 2010; Boddey et al., 2010; Tarr et al., 2013; Osborne et al., 2010). However, the protease cleavage of plasmepsin V does not appear to be the sole deciding factor for translocation into the host cells as $\mathrm{PI}(3) \mathrm{P}$ binding to the effector proteins in the ER upstream of the PEXEL motif seems to be a prerequisite step for translocation in addition to the plasmepsin V cleavage (Bhattacharjee et al., 2012a; Bhattacharjee et al., 2012b; Bhattacharjee et al., 2012c; Haldar et al., 2016; Hsiao et al., 2013). Another class of effector proteins, PEXEL negative exported proteins (PNEPs), do not contain PEXEL motif but still can translocate to the host cells (Pachlatko et al., 2010; Bhattacharjee et al., 2012a; Spycher et al., 2006; Saridaki et al., 2009; Haase et al., 2009; Heiber et al., 2013; Jani et al., 2008). The first 20 amino acids at the $\mathrm{N}$ terminus of PNEPs without a classical ' $\mathrm{N}$ ' terminal signal sequence are sufficient to export non PEXEL effector proteins to the host cells (Saridaki et al., 2009; Haase et al., 2009) indicating a general export property of PNEPs. However, effector proteins of both the classes, PEXEL dependent and independent, do unfold in the vacuolar space before they traverse through the PTEX complex (Grüring et al., 2012; Desai et al., 2014b; Ho et al., 2018; Garten et al., 2018).

The process of oligomerization of $\mathrm{P} 2$ in the parasite cytoplasm precedes the localization on the iRBC surface. Out of several oligomeric species in the parasite cytoplasm at the trophozoite stage, only homo/hetero tetrameric species of P2 appear to translocate to the iRBC surface through iRBC cytoplasm (Das et al., 2012a). Neither ghost from iRBCs surface nor the iRBCs cytoplasm showed the presence of monomeric P2 indicating that the oligomerization in the parasite cytoplasm could be initial one of the prerequisite steps in the export mechanism of P2. In the 48h life cycle in iRBCs, the oligomerization window of P2 in the parasite cytoplasm approximately starts at around 22-24h post merozoite invasion (PMI) and continues till 34-36h PMI at late trophozoite to the early schizogonic stage (Das et al., 2012a). Before 22-24h and after 36-38h PMI, there were no oligomeric species of P2 observed in the western blot experiment (Das et al., 2012a; Das et al., 2012b) indicating tight regulation of oligomerization in that window of time and a possible link between oligomerization and export. After that window, oligomerization diminishes possibly due to the secretion of P2 into the culture supernatant or digestion. Immunofluorescence assay (IFA) using E2G12 after 34-36h PMI does not seem to suggest a possibility of P2 recycling back to the parasite from the host cells as iRBC cytosol did not show any P2 staining (Das et al., 2012a). 
P2 does not contain any PEXEL motif and also it is devoid of classical export signal hence it comes under the PNEP class and possibly translocate through an unknown pathway. Transient transfection with P2-GFP followed by immunoblotting does not seem to suggest any protease processing of P2-GFP before export into the host cells as the molecular mass of P2-GFP did not deviate from the theoretical mass (Das et al., 2012a). In addition to oligomerization, the probable route of $\mathrm{P} 2$ translocation might have been PI(3)P binding and unfolding of the P2 tetramer/oligomer before PTEX traversing.

\section{In solution, P2 forms a stable tetramer which is a molten globule in nature having hydro- phobic pockets on the surface}

NMR experiments in solution distinctly revealed that recombinant P2 protein has a high propensity to oligomerize and tend to form aggregates by self-association at a millimolar concentration (Mishra et al., 2012; Mishra et al., 2014a). Further exploration using circular dichroism (CD) and solution NMR has also revealed that the recombinant monomeric species of $\mathrm{P} 2$ is predominantly $\alpha$ helical but molten globule in nature and the ' $\mathrm{C}$ ' terminal region is intrinsically disordered in structure (Mishra et al., 2014b, Mishra et al., 2015). At physiological $\mathrm{pH}$ 7.4, the thermodynamic stability of the monomer shifts towards tetramerization maintaining the molten globule nature of each monomer in the tetramer (Mishra et al., 2015), thereby keeping the flexibility of the entire tetrameric complex. Urea denaturation of recombinant P2 followed by residue level interrogation using NMR further revealed that two monomeric P2 molecules associate to form a dimer and two such dimer molecules packaged closely at their $\mathrm{N}$ terminus having $\alpha$ helices to form the tetramer (Mishra et al., 2015). 2D ${ }^{1} \mathrm{H}-{ }^{15} \mathrm{~N}$ HSQC spectra of the native deuterated tetrameric form of $\mathrm{P} 2$ exhibited hydrophobic surface/pocket mostly contributed by the $\mathrm{N}$ terminal $\alpha$ helices as shown using 8-anilinonaphthalene-1-sulfonic acid (ANS) (Mishra et al., 2015) indicating that P2 tetramer could provide sites for intermolecular association in an aqueous environment and may have the possibility to bind non-polar molecules in its natively localized environment, i.e., iRBCs surface. Between P. falciparumP2 and Human $\mathrm{P} 2$, there is $69 \%$ amino acid sequence homology but they differ in their oligomerization pattern and behavior as Human P2 at physiological pH forms a stable dimer (ref) but in the same conditionP. falciparum P2 forms a molten globule tetramer indicating some functional implications of tetrameric P2 on the surface of iRBCs at late trophozoite stage. In a recent discovery, it has been demonstrated that P2 tetramers stabilize themselves on the iRBC surface by interacting with RBC Band3 protein where $\mathrm{N}$ terminal 70 amino acids of P2 interact to form the oligomers and associate with Band 3 protein (Mishra et al., 2020).

\section{What could be the possible function of oligomeric/tetrameric P2 on the iRBC surface?}

The selectivity of the infected red blood cell membrane is compromised due to the translocation of several effector proteins into the host cells. Virulence factors and channel proteins both are predominant components of the exportome which plays important role in disease biology and solute uptake respectively. After the erythrocyte invasion by malaria parasites, induction of a broad specificity channel known as the new permeability pathway (NPP) into the host cells renders the red blood cell membrane non-selective and promiscuous for a range of small molecule solutes including ions (Kirk et al., 2015). Blockage of NPP by furosemide resulted in the pronounced inhibition of parasite growth in culture suggesting the indispensability of NPP in iRBCs (Staines et al., 2004). One of the channel molecules of NPP has recently been extensively characterized as a plasmodial surface anion channel (PSAC), a widely accepted iRBC channel protein shown to enhance nutrient permeability of iRBCs (Kirk et al., 2015; Alkhalil et al., 2009, Desai et al., 2012). While the enhanced permeability of iRBCs has been experimented out for decades, the molecular identity of PSAC was largely unknown until recently a cytoadherence-linked antigen 3 (Clag3) is the key player of the channel for nutrient uptake (Alkhalil et al., 2009, Desai et al., 2012; Desai et al., 2014; Nguitragool et al., 2014; Gupta et al., 2018). In the formation of PSAC, Clag3 protein forms a homodimer, and RhopH2 and RhopH3 do associate with the dimer for the construction of functional channel (Nguitragool et al., 2014; Gupta et al., 2018; Kaneko et al., 2005; Ito et al., 2017; Sherling et al., 2017; Counihan et al., 2017; Schureck et al., 2021). Under PTEX suppressed condition, Clag3 still translocate into the host cell suggesting an alternative mechanism of Clag3 export but in the PTEX suppressed parasite lines, the transport of solutes by PSAC was diminished (Beck et al., 2014; Comeaux et al., 2011) indicating that other exported proteins are required 
for channel formation either in association with or independent of Clag3.

Based on experimental evidence, the non-ribosomal role (s) of oligomeric/tetrameric P2 on the iRBC surface appears to be important but currently is at the stage of speculation. The tight regulation of oligomerization and subsequent localization of oligomer/tetramer on the iRBC surface at the trophozoite stage drives the attention towards its direct possible role in the formation of some channel either in association with Clag3 or independently for small molecule transport. P2 has one putative transmembrane (TM) domain from amino acid N'64-84C' but in the oligomeric state how this TM domain is important for host membrane insertion is currently elusive. Immunofluorescence assay (IFA) at mid to late trophozoite stage showed the presence of Clag3 on iRBC surface co-localized with RhopH3 (Sherling et al., 2017). But at this stage of mid to late trophozoite, P2 oligomers were diminished as IFA using E2G12 did not stain the iRBC surface suggesting that oligomeric P2 may not be a component of PSAC but there is a possibility of channel formation by P2 oligomers either in association with other export proteins or independently. If oligomeric P2 is forming a channel with/without other parasitic proteins then this channel does not seem to complement the function of PSAC under the null state of Clag3.1 and Clag3.2 as these null parasites showed significant growth inhibition (Nguitragool et al., 2014; Gupta et al., 2018; Kaneko et al., 2005), hence there is a possibility that PSAC and putative channel of oligomeric P2 both are working independently.

Oligomeric/tetrameric P2 being molten globule in nature and having hydrophobic pockets on the surface also drives the speculation towards its possible direct interaction with hydrophobic molecules such as lipids. Parasite culture medium devoid of oleic acid and palmitic acid resulted in parasite cell cycle arrest (Mitamura et al., 2000) similar to P2 antibody-mediated arrest (Das et al., 2012a). Hence it could be logical to think and rationale to design experiments to validate the interaction of $\mathrm{P} 2$ oligomers with lipids which are already reported to be crucial for parasite progression. In addition to possible channel formation or P2-lipid interaction, oligomeric P2 might have other non-ribosomal function (s) depending on the interaction of P2 with other partner proteins on the iRBC surface and the structure of the entire oligomeric complex.

\section{Frontier questions}

Finding oligomeric parasite P2 protein on the iRBC surface was puzzling but at the same time raises the possibility to target it. Unraveling the function of P2 on the iRBCs surface could open several avenues towards our effort to develop novel anti-malarial small molecules and might also come under consideration as a possible vaccine candidate as recently reported (Szuster-Ciesielska et al., 2019). Understanding a fundamental non-ribosomal role (s) of a ribosomal protein in the propagation of Plasmodium parasite in red blood cells can be harnessed to develop new strategies to target this highly resilient parasite and will unmask novel biochemical pathways operational at the trophozoite stage of parasite propagation. Based on current understanding, below there are three key unresolved questions depicted in Figure 1 that need to be answered to understand the eccentric P2 cell biology in Plasmodium parasites, and towards that, my laboratory is fully engaged.

What is the function (s) of oligomeric/tetrameric P2 on the iRBC surface at the late trophozoite/early schizogonic stage?

What is the mechanism of P2 translocation from the parasite cytoplasm to the iRBC surface?

What is the nature of P2 oligomers on the iRBC surface? Does it form a channel complex?

\section{Conclusions}

Cell division checkpoints and non-PEXEL protein export in Plasmodium falciparum-infected human RBCs are the two aspects that need to be pondered in apicomplexan cell biology. In this review, we have summarised the uncanny nature of a parasite ribosomal stalk protein P2 which appears to translocate to the infected RBC (iRBC) surface and seems to be involved in the regulation of parasite nuclear division at the initial stage. P2 being a non-PEXEL protein, requires prior oligomerization before trafficking to the iRBC surface which indicates that for non-PEXEL protein export, oligomerization might be one of the prerequisite steps in the cascade of molecular events of non-PEXEL protein trafficking in iRBCs. 
Functional inhibition of P2 oligomers on the iRBC surface either by using monoclonal antibodies or by genetic knockdown resulted in the nuclear division arrest of the parasites which possibly suggests that the P2 oligomers might be involved as a checkpoint regulator or simply as a gatekeeper at the initial stage of nuclear division. Oligomeric P2 on the iRBC surface appears to form a channel protein complex either independently or in association with other parasite proteins to perform a regulatory role during parasite nuclear division. These serendipitous findings of the uncanny nature of $\mathrm{P} 2$ protein in malaria parasites offer to explore more about non-ribosomal indefensible functions of ribosomal proteins in apicomplexan parasites and certainly, the molecular role of P2 oligomers in the regulation of parasite nuclear division are being looked at towards a better fundamental understanding about cell division checkpoints and the possibility to inhibit the function of $\mathrm{P} 2$ oligomers using small molecules in iRBCs.

\section{Data availability statement}

Not applicable.

\section{Author contributions}

Sudipta Das- Conceptualization (lead); writing-original draft(lead); review and editing(lead), Bhaskar RoyWriting-original draft(supporting); review and editing(supporting), Saswata Chakrabarty- Writing-original draft(supporting); review and editing(supporting). All authors have read and agreed to the published version of this manuscript.

\section{Acknowledgments}

Dr. Sudipta Das's laboratory has been funded by Ramalingaswami Fellowship, Department of Biotechnology (DBT), Govt. of India; Core Research Grant, Department of Science and Technology (DST), Govt. of India and CSIR, Govt. of India.

\section{Conflicts of interests}

None declared

\section{Ethics statement}

None required.

\section{References}

Alkhalil, A., Pillai, A. D., Bokhari, A. A., Vaidya, A. B., \& Desai, S. A. (2009). Complex inheritance of the plasmodial surface anion channel in a Plasmodium falciparum genetic cross. Mol Microbiol, 72 (2), 459-469. doi:10.1111/j.1365-2958.2009.06661.x

Beck, J. R., Muralidharan, V., Oksman, A., \& Goldberg, D. E. (2014). PTEX component HSP101 mediates export of diverse malaria effectors into host erythrocytes. Nature, 511 (7511), 592-595. doi:10.1038/nature13574

Bhattacharjee, S., Speicher, K. D., Stahelin, R. V., Speicher, D. W., \& Haldar, K. (2012a). $\mathrm{PI}(3) \mathrm{P}$-independent and -dependent pathways function together in a vacuolar translocation sequence to target malarial proteins to the host erythrocyte. Mol Biochem Parasitol, 185 (2), 106-113. doi:10.1016/j.molbiopara.2012.07.004

Bhattacharjee, S., Stahelin, R. V., \& Haldar, K. (2012b). Host targeting of virulence determinants and phosphoinositides in blood stage malaria parasites. Trends Parasitol, 28 (12), 555-562. doi:10.1016/j.pt.2012.09.004

Bhattacharjee, S., Stahelin, R. V., Speicher, K. D., Speicher, D. W., \& Haldar, K. (2012c). Endoplasmic reticulum PI(3)P lipid binding targets malaria proteins to the host cell. Cell, 148 (1-2), 201-212. doi:10.1016/j.cell.2011.10.051 
Boddey, J. A., Hodder, A. N., Gunther, S., Gilson, P. R., Patsiouras, H., Kapp, E. A., . . . Cowman, A. F. (2010). An aspartyl protease directs malaria effector proteins to the host cell. Nature, 463 (7281), 627-631. doi:10.1038/nature08728

Bushell, E., Gomes, A. R., Sanderson, T., Anar, B., Girling, G., Herd, C., . . . Billker, O. (2017). Functional Profiling of a Plasmodium Genome Reveals an Abundance of Essential Genes. Cell, 170 (2), 260-272 e268. doi:10.1016/j.cell.2017.06.030

Chatterjee, S., Singh, S., Sohoni, R., Singh, N. J., Vaidya, A., Long, C., \& Sharma, S. (2000). Antibodies against ribosomal phosphoprotein $\mathrm{P} 0$ of Plasmodium falciparum protect mice against challenge with Plasmodium yoelii. Infect Immun, 68 (7), 4312-4318. doi:10.1128/iai.68.7.4312-4318.2000

Comeaux, C. A., Coleman, B. I., Bei, A. K., Whitehurst, N., \& Duraisingh, M. T. (2011). Functional analysis of epigenetic regulation of tandem RhopH1/clag genes reveals a role in Plasmodium falciparum growth. Mol Microbiol, 80 (2), 378-390. doi:10.1111/j.1365-2958.2011.07572.x

Counihan, N. A., Chisholm, S. A., Bullen, H. E., Srivastava, A., Sanders, P. R., Jonsdottir, T. K., . . . de Koning-Ward, T. F. (2017). Plasmodium falciparum parasites deploy RhopH2 into the host erythrocyte to obtain nutrients, grow and replicate. Elife, 6 . doi:10.7554/eLife.23217

Das, S., Basu, H., Korde, R., Tewari, R., \& Sharma, S. (2012a). Arrest of nuclear division in Plasmodium through blockage of erythrocyte surface exposed ribosomal protein P2. PLoS Pathog, 8 (8), e1002858. doi:10.1371/journal.ppat.1002858

Das, S., Sudarsan, R., Sivakami, S., \& Sharma, S. (2012b). Erythrocytic stage-dependent regulation of oligomerization of Plasmodium ribosomal protein P2. J Biol Chem, 287 (49), 41499-41513. doi:10.1074/jbc.M112.384388

Desai, S. A. (2012). Ion and nutrient uptake by malaria parasite-infected erythrocytes. Cell Microbiol, 14 (7), 1003-1009. doi:10.1111/j.1462-5822.2012.01790.x

Desai, S. A. (2014a). Why do malaria parasites increase host erythrocyte permeability? Trends Parasitol, 30 (3), 151-159. doi:10.1016/j.pt.2014.01.003

Desai, S. A., \& Miller, L. H. (2014b). Malaria: Protein-export pathway illuminated. Nature, 511 (7511), 541-542. doi:10.1038/nature13646

Diaconu, M., Kothe, U., Schlunzen, F., Fischer, N., Harms, J. M., Tonevitsky, A. G., . . . Wahl, M. C. (2005). Structural basis for the function of the ribosomal L7/12 stalk in factor binding and GTPase activation. Cell, 121 (7), 991-1004. doi:10.1016/j.cell.2005.04.015

Francisco-Velilla, R., \& Remacha, M. (2010). In vivo formation of a stable pentameric (P2alpha/P1beta)P0-(P1alpha/P2beta) ribosomal stalk complex in Saccharomyces cerevisiae. Yeast, 27 (9), 693-704. doi:10.1002/yea. 1765

Garten, M., Nasamu, A. S., Niles, J. C., Zimmerberg, J., Goldberg, D. E., \& Beck, J. R. (2018). EXP2 is a nutrient-permeable channel in the vacuolar membrane of Plasmodium and is essential for protein export via PTEX. Nat Microbiol, 3 (10), 1090-1098. doi:10.1038/s41564-018-0222-7

Goldberg, D. E., \& Cowman, A. F. (2010). Moving in and renovating: exporting proteins from Plasmodium into host erythrocytes. Nat Rev Microbiol, 8 (9), 617-621. doi:10.1038/nrmicro2420

Gonzalo, P., \& Reboud, J. P. (2003). The puzzling lateral flexible stalk of the ribosome. Biol Cell, 95 (3-4), 179-193. doi:10.1016/s0248-4900(03)00034-0

Goswami, A., Singh, S., Redkar, V. D., \& Sharma, S. (1997). Characterization of P0, a ribosomal phosphoprotein of Plasmodium falciparum. Antibody against amino-terminal domain inhibits parasite growth. $J$ Biol Chem, 272 (18), 12138-12143. doi:10.1074/jbc.272.18.12138 
Grela, P., Li, XP., Horbowicz, P. et al. (2017). Human ribosomal P1-P2 heterodimer represents an optimal docking site for ricin A chain with a prominent role for P1 C-terminus. Sci

Rep 7, 5608 . doi.org/10.1038/s41598-017-05675-5

Gruring, C., Heiber, A., Kruse, F., Flemming, S., Franci, G., Colombo, S. F., . . . Spielmann, T. (2012). Uncovering common principles in protein export of malaria parasites. Cell Host Microbe, 12 (5), 717-729. doi:10.1016/j.chom.2012.09.010

Gupta, A., Balabaskaran-Nina, P., Nguitragool, W., Saggu, G. S., Schureck, M. A., \& Desai, S. A. (2018). CLAG3 Self-Associates in Malaria Parasites and Quantitatively Determines Nutrient Uptake Channels at the Host Membrane. mBio, 9 (3). doi:10.1128/mBio.02293-17

Haase, S., Herrmann, S., Gruring, C., Heiber, A., Jansen, P. W., Langer, C., . . . Spielmann, T. (2009). Sequence requirements for the export of the Plasmodium falciparum Maurer's clefts protein REX2. Mol Microbiol, 71 (4), 1003-1017. doi:10.1111/j.1365-2958.2008.06582.x

Haldar, K. $(2016)^{\mathrm{b}}$. Protein trafficking in apicomplexan parasites: crossing the vacuolar Rubicon. Curr Opin Microbiol, 32 , 38-45. doi:10.1016/j.mib.2016.04.013

Haldar, K., Samuel, B. U., Mohandas, N., Harrison, T., \& Hiller, N. L. (2001)a . Transport mechanisms in Plasmodium-infected erythrocytes: lipid rafts and a tubovesicular network. Int J Parasitol, 31 (12), 1393-1401. doi:10.1016/s0020-7519(01)00251-x

Hanson, C. L., Videler, H., Santos, C., Ballesta, J. P., \& Robinson, C. V. (2004). Mass spectrometry of ribosomes from Saccharomyces cerevisiae: implications for assembly of the stalk complex. J Biol Chem, 279 (41), 42750-42757. doi:10.1074/jbc.M405718200

Heiber, A., Kruse, F., Pick, C., Gruring, C., Flemming, S., Oberli, A., . . . Spielmann, T. (2013). Identification of new PNEPs indicates a substantial non-PEXEL exportome and underpins common features in Plasmodium falciparum protein export. PLoS Pathog, 9 (8), e1003546. doi:10.1371/journal.ppat.1003546

Hiller, N. L., Bhattacharjee, S., van Ooij, C., Liolios, K., Harrison, T., Lopez-Estrano, C., \& Haldar, K. (2004). A host-targeting signal in virulence proteins reveals a secretome in malarial infection.Science, 306 (5703), 1934-1937. doi:10.1126/science.1102737

Ho, C. M., Beck, J. R., Lai, M., Cui, Y., Goldberg, D. E., Egea, P. F., \& Zhou, Z. H. (2018). Malaria parasite translocon structure and mechanism of effector export. Nature, 561 (7721), 70-75. doi:10.1038/s41586-0180469-4

Hsiao, C. H., Luisa Hiller, N., Haldar, K., \& Knoll, L. J. (2013). A HT/PEXEL motif in Toxoplasma dense granule proteins is a signal for protein cleavage but not export into the host cell. Traffic, 14 (5), 519-531. doi:10.1111/tra.12049

Ito, D., Schureck, M. A., \& Desai, S. A. (2017). An essential dual-function complex mediates erythrocyte invasion and channel-mediated nutrient uptake in malaria parasites. Elife, 6 . doi:10.7554/eLife.23485

Jani, D., Nagarkatti, R., Beatty, W., Angel, R., Slebodnick, C., Andersen, J., . . . Rathore, D. (2008). HDP-a novel heme detoxification protein from the malaria parasite. PLoS Pathog, 4 (4), e1000053. doi:10.1371/journal.ppat.1000053

Jimenez-Diaz, A., Remacha, M., Ballesta, J. P., \& Berlanga, J. J. (2013). Phosphorylation of initiation factor eIF2 in response to stress conditions is mediated by acidic ribosomal P1/P2 proteins in Saccharomyces cerevisiae. PLoS One, 8 (12), e84219. doi:10.1371/journal.pone.0084219

Kaneko, O., Yim Lim, B. Y., Iriko, H., Ling, I. T., Otsuki, H., Grainger, M., . . . Torii, M. (2005). Apical expression of three RhopH1/Clag proteins as components of the Plasmodium falciparum RhopH complex. Mol Biochem Parasitol, 143 (1), 20-28. doi:10.1016/j.molbiopara.2005.05.003 
Kang, C. H., Lee, Y. M., Park, J. H., Nawkar, G. M., Oh, H. T., Kim, M. G., . . . Lee, S. Y. (2016). Ribosomal P3 protein AtP3B of Arabidopsis acts as both protein and RNA chaperone to increase tolerance of heat and cold stresses. Plant Cell Environ, 39 (7), 1631-1642. doi:10.1111/pce.12742

Kirk, K. (2015). Ion Regulation in the Malaria Parasite. Annu Rev Microbiol, 69 , 341-359. doi:10.1146/annurev-micro-091014-104506

Lauer, S. A., Rathod, P. K., Ghori, N., \& Haldar, K. (1997). A membrane network for nutrient import in red cells infected with the malaria parasite. Science, $2^{76}$ (5315), 1122-1125. doi:10.1126/science.276.5315.1122

Lobo, C. A., Kar, S. K., Ravindran, B., Kabilan, L., \& Sharma, S. (1994). Novel proteins of Plasmodium falciparum identified by differential immunoscreening using immune and patient sera. Infect Immun, 62 (2), 651-656. doi:10.1128/IAI.62.2.651-656.1994

Marti, M., Good, R. T., Rug, M., Knuepfer, E., \& Cowman, A. F. (2004). Targeting malaria virulence and remodeling proteins to the host erythrocyte. Science, 306 (5703), 1930-1933. doi:10.1126/science.1102452

Martinez-Azorin, F., Remacha, M., \& Ballesta, J. P. (2008). Functional characterization of ribosomal P1/P2 proteins in human cells.Biochem J, 413 (3), 527-534. doi:10.1042/BJ20080049

Mishra, P., Choudhary, S., Mukherjee, S., Sengupta, D., Sharma, S., \& Hosur, R. V. (2015). Molten globule nature of Plasmodium falciparum P2 homo-tetramer. Biochem Biophys Rep, 1 , 97-107. doi:10.1016/j.bbrep.2015.03.010

Mishra, P., Das, S., Panicker, L., Hosur, M. V., Sharma, S., \& Hosur, R. V. (2012). NMR insights into folding and self-association of Plasmodium falciparum P2. PLoS One, 7 (5), e36279. doi:10.1371/journal.pone.0036279

Mishra, P., Dmello, C., Sengupta, D., Chandrabhan Singh, S., Kirkise, N., Hosur, R. V., \& Sharma, S. (2020). Molecular study of binding of Plasmodium ribosomal protein P2 to erythrocytes. Biochimie, 176 , 181-191. doi:10.1016/j.biochi.2020.07.007

Mishra, P., Rajagopal, S., Sharma, S., \& Hosur, R. V. (2014a). The C-terminal domain of eukaryotic acidic ribosomal P2 proteins is intrinsically disordered with conserved structural propensities.Protein Pept Lett, 22 (3), 212-218. doi:10.2174/0929866521666141121160523

Mishra, P., Sharma, S., \& Hosur, R. V. (2014b). Residue level description of in vivo self-association of Plasmodium falciparum P2.J Biomol Struct Dyn, 32 (4), 602-612. doi:10.1080/07391102.2013.782827

Mitamura, T., Hanada, K., Ko-Mitamura, E. P., Nishijima, M., \& Horii, T. (2000). Serum factors governing intraerythrocytic development and cell cycle progression of Plasmodium falciparum. Parasitol Int, 49 (3), 219-229. doi:10.1016/s1383-5769(00)00048-9

Nguitragool, W., Bokhari, A. A., Pillai, A. D., Rayavara, K., Sharma, P., Turpin, B., . . . Desai, S. A. (2011). Malaria parasite clog genes determine channel-mediated nutrient uptake by infected red blood cells. Cell, 145 (5), 665-677. doi:10.1016/j.cell.2011.05.002

Nusspaumer, G., Remacha, M., \& Ballesta, J. P. (2000). Phosphorylation and N-terminal region of yeast ribosomal protein P1 mediate its degradation, which is prevented by protein P2. EMBO J, 19 (22), 60756084. doi:10.1093/emboj/19.22.6075

Osborne, A. R., Speicher, K. D., Tamez, P. A., Bhattacharjee, S., Speicher, D. W., \& Haldar, K. (2010). The host targeting motif in exported Plasmodium proteins is cleaved in the parasite endoplasmic reticulum. Mol Biochem Parasitol, 171 (1), 25-31. doi:10.1016/j.molbiopara.2010.01.003

Pachlatko, E., Rusch, S., Muller, A., Hemphill, A., Tilley, L., Hanssen, E., \& Beck, H. P. (2010). MAHRP2, an exported protein of Plasmodium falciparum, is an essential component of Maurer's cleft tethers. Mol Microbiol, 77 (5), 1136-1152. doi:10.1111/j.1365-2958.2010.07278.x 
Remacha, M., Jimenez-Diaz, A., Santos, C., Briones, E., Zambrano, R., Rodriguez Gabriel, M. A., . . . Ballesta, J. P. (1995). Proteins P1, P2, and P0, components of the eukaryotic ribosome stalk. New structural and functional aspects. Biochem Cell Biol, 73 (11-12), 959-968. doi:10.1139/o95-103

Remacha, M., Santos, C., Bermejo, B., Naranda, T., \& Ballesta, J. P. (1992). Stable binding of the eukaryotic acidic phosphoproteins to the ribosome is not an absolute requirement for in vivo protein synthesis. $J$ Biol Chem, 267 (17), 12061-12067. Retrieved from https://www.ncbi.nlm.nih.gov/pubmed/1601875

Rodriguez-Mateos, M., Garcia-Gomez, J. J., Francisco-Velilla, R., Remacha, M., de la Cruz, J., \& Ballesta, J. P. (2009). Role and dynamics of the ribosomal protein P0 and its related trans-acting factor Mrt4 during ribosome assembly in Saccharomyces cerevisiae. Nucleic Acids Res, 37 (22), 7519-7532. doi:10.1093/nar/gkp806

Russo, I., Babbitt, S., Muralidharan, V., Butler, T., Oksman, A., \& Goldberg, D. E. (2010). Plasmepsin V licenses Plasmodium proteins for export into the host erythrocyte. Nature, 463 (7281), 632-636. doi:10.1038/nature08726

Santos, C., \& Ballesta, J. P. (1994)a . Ribosomal protein P0, contrary to phosphoproteins P1 and P2, is required for ribosome activity and Saccharomyces cerevisiae viability. J Biol Chem, 269 (22), 15689-15696. Retrieved from https://www.ncbi.nlm.nih.gov/pubmed/8195220

Santos, C., \& Ballesta, J. P. $(1995)^{\mathrm{b}}$. The highly conserved protein P0 carboxyl end is essential for ribosome activity only in the absence of proteins P1 and P2. J Biol Chem, 270 (35), 20608-20614. doi:10.1074/jbc.270.35.20608

Saridaki, T., Frohlich, K. S., Braun-Breton, C., \& Lanzer, M. (2009). Export of PfSBP1 to the Plasmodium falciparum Maurer's clefts. Traffic, 10 (2), 137-152. doi:10.1111/j.1600-0854.2008.00860.x

Sherling, E. S., Knuepfer, E., Brzostowski, J. A., Miller, L. H., Blackman, M. J., \& van Ooij, C. (2017). The Plasmodium falciparum rhoptry protein RhopH3 plays essential roles in host cell invasion and nutrient uptake. Elife, 6 . doi:10.7554/eLife.23239

Singh, S., Sehgal, A., Waghmare, S., Chakraborty, T., Goswami, A., \& Sharma, S. (2002). Surface expression of the conserved ribosomal protein P0 on parasite and other cells. Mol Biochem Parasitol, 119 (1), 121-124. doi:10.1016/s0166-6851(01)00394-2

Spillman, N. J., Beck, J. R., \& Goldberg, D. E. (2015). Protein export into malaria parasite-infected erythrocytes: mechanisms and functional consequences. Annu Rev Biochem, 84 , 813-841. doi:10.1146/annurevbiochem-060614-034157

Spycher, C., Rug, M., Klonis, N., Ferguson, D. J., Cowman, A. F., Beck, H. P., \& Tilley, L. (2006). Genesis of and trafficking to the Maurer's clefts of Plasmodium falciparum-infected erythrocytes. Mol Cell Biol, 26 (11), 4074-4085. doi:10.1128/MCB.00095-06

Staines, H. M., Dee, B. C., O’Brien, M., Lang, H. J., Englert, H., Horner, H. A., . . . Kirk, K. (2004). Furosemide analogues as potent inhibitors of the new permeability pathways of Plasmodium falciparum-infected human erythrocytes. Mol Biochem Parasitol, 133 (2), 315-318. doi:10.1016/j.molbiopara.2003.10.009

Sudarsan, R., Chopra, R. K., Khan, M. A., \& Sharma, S. (2015). Ribosomal protein P2 localizes to the parasite zoite-surface and is a target for invasion inhibitory antibodies in Toxoplasma gondii and Plasmodium falciparum. Parasitol Int, 64 (1), 43-49. doi:10.1016/j.parint.2014.08.006

Szuster-Ciesielska, A., Wawiorka, L., Krokowski, D., Grankowski, N., Jarosz, L., Lisiecka, U., \& Tchorzewski, M. (2019). Immunogenic Evaluation of Ribosomal P-Protein Antigen P0, P1, and P2 and Pentameric Protein Complex P0-(P1-P2)2 of Plasmodium falciparum in a Mouse Model.J Immunol Res, 2019, 9264217. doi: $10.1155 / 2019 / 9264217$ 
Tamez, P. A., Bhattacharjee, S., van Ooij, C., Hiller, N. L., Llinas, M., Balu, B., . . . Haldar, K. (2008). An erythrocyte vesicle protein exported by the malaria parasite promotes tubovesicular lipid import from the host cell surface. PLoS Pathog, \& (8), e1000118. doi:10.1371/journal.ppat.1000118

Tarr, S. J., Cryar, A., Thalassinos, K., Haldar, K., \& Osborne, A. R. (2013). The C-terminal portion of the cleaved HT motif is necessary and sufficient to mediate export of proteins from the malaria parasite into its host cell. Mol Microbiol, 87 (4), 835-850. doi:10.1111/mmi.12133

Tchorzewski, M., Krokowski, D., Rzeski, W., Issinger, O. G., \& Grankowski, N. (2003). The subcellular distribution of the human ribosomal "stalk" components: P1, P2 and P0 proteins. Int J Biochem Cell Biol, 35 (2), 203-211. doi:10.1016/s1357-2725(02)00133-4

Uchiumi, T., \& Kominami, R. (1992). Direct evidence for interaction of the conserved GTPase domain within 28 S RNA with mammalian ribosomal acidic phosphoproteins and L12. J Biol Chem, 267 (27), 19179-19185. Retrieved from https://www.ncbi.nlm.nih.gov/pubmed/1527039

Volarevic, S., Stewart, M. J., Ledermann, B., Zilberman, F., Terracciano, L., Montini, E., . . . Thomas, G. (2000). Proliferation, but not growth, blocked by conditional deletion of 40S ribosomal protein S6. Science, 288 (5473), 2045-2047. doi:10.1126/science.288.5473.2045

Wan, F., Anderson, D. E., Barnitz, R. A., Snow, A., Bidere, N., Zheng, L., . . . Lenardo, M. J. (2007). Ribosomal protein S3: a KH domain subunit in NF-kappaB complexes that mediates selective gene regulation.Cell, 131 (5), 927-939. doi:10.1016/j.cell.2007.10.009

Wool, I. G. (1996). Extraribosomal functions of ribosomal proteins. Trends Biochem Sci, 21 (5), 164-165. Retrieved from https://www.ncbi.nlm.nih.gov/pubmed/8871397

Zhang, M., Wang, C., Otto, T. D., Oberstaller, J., Liao, X., Adapa, S. R., . . . Adams, J. H. (2018). Uncovering the essential genes of the human malaria parasite Plasmodium falciparum by saturation mutagenesis.Science, 360 (6388). doi:10.1126/science.aap7847

Zinker, S., \& Warner, J. R. (1976). The ribosomal proteins of Saccharomyces cerevisiae. Phosphorylated and exchangeable proteins.J Biol Chem, 251 (6), 1799-1807. Retrieved from https://www.ncbi.nlm.nih.gov/pubmed/767341

\section{Figure Legend}

Fig. 1 Cartoon of P. Falciparum infected trophozoite stage iRBC depicting oligomeric states and localization of ribosomal P2 protein in different compartments of iRBC raising several critical questions portrayed in the diagram. PN: Parasite Nucleus; PC: Parasite cytoplasm; RRBC: Red Blood Cell Cytoplasm; RBCM: Red Blood Cell Membrane. 
P. falciparum trophozoite stage ( $30 \mathrm{~h})$ iRBC

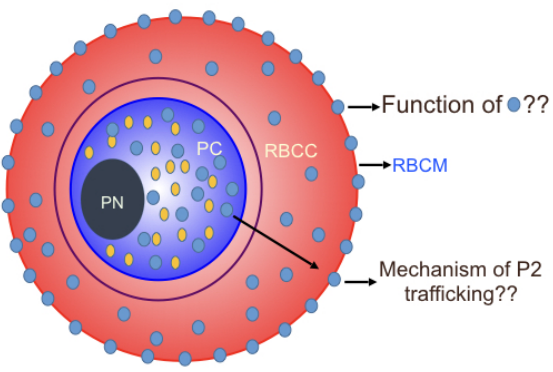

- Tetrameric or P2 o Monomeric P2 higher oligomers

PN: Parasite nucleus PC: Parasite cytoplasm

RBCC: Red Blood cell Cytoplasm

RBCM: Red Blood Cell Membrane

Figure. 1 